Supporting information

\title{
Constructing Heterogeneous Structure in Metal-Organic Framework-Derived Hierarchical Sulfur Hosts for Capturing Polysulfides and Promoting Conversion Kinetics
}

Yingbo Xiao ${ }^{l}$, Sijia Guo ${ }^{l}$, Yuan Ouyang ${ }^{l}$, Dixiong $\mathrm{Li}^{l}$, Xin $\mathrm{Li}^{l}$, Wenchao He ${ }^{l}$, Haoyan Deng $^{l}$, Wei Gong ${ }^{l}$, Chao Tan ${ }^{l}$, Qinghan Zeng ${ }^{l}$, Qi Zhang ${ }^{1,2 *}$ and Shaoming

$$
\text { Huang }^{1,2 *}
$$

${ }^{1}$ Guangzhou Key Laboratory of Low-Dimensional Materials and Energy Storage

Devices

School of Materials and Energy, Guangdong University of Technology, Guangzhou, 510006, China

${ }^{2}$ Synergy Innovation Institute of GDUT, Heyuan, 517000, China.

E-mail: qzhangmse@ gdut.edu.cn

E-mail: smhuang@gdut.edu.cn 


\section{EXPERIMENTAL SECTION}

Chemical Reagents and Materials. Graphene oxide (GO) and carbon nanotubes (CNTs) were obtained from Nanjing XFNANO Material Technology Co., Ltd (China). Carbon paper was purchased from Hesen Electric Inc. Shanghai. Lithium-metal foil was obtained from Tianjin Zhongneng lithium Co. Ltd (China). Other reagents, unless otherwise stated, were obtained from commercial sources (Sigma Aldrich, Alfa Aesar) and were used without further purification.

Synthesis of $\mathrm{Bi}_{2} \mathrm{O}_{3} @ \mathrm{C} @ \mathrm{G}-\mathrm{S}, \mathrm{Bi} / \mathrm{Bi}_{2} \mathrm{O}_{3} @ \mathrm{C} @ \mathrm{G}-\mathrm{S}$, and $\mathrm{Bi} @ \mathrm{C} @ \mathrm{G}-\mathrm{S}$ composites. The composites were obtained by the melting-diffusion method. $\mathrm{Bi}_{2} \mathrm{O}_{3} @ \mathrm{C} @ \mathrm{G}$, Bi/ $/ \mathrm{Bi}_{2} \mathrm{O}_{3} @ \mathrm{C} @ \mathrm{G}$, or Bi@C@G was milled with sulfur uniformly with a mass radio of 1:3 and heated at $155^{\circ} \mathrm{C}$ for $12 \mathrm{~h}$ in an airtight Teflon-lined stainless steel autoclave to obtain $\mathrm{Bi}_{2} \mathrm{O}_{3} @ \mathrm{C} @ \mathrm{G}-\mathrm{S}, \mathrm{Bi} / \mathrm{Bi}_{2} \mathrm{O}_{3} @ \mathrm{C} @ \mathrm{G}-\mathrm{S}$, and $\mathrm{Bi} @ \mathrm{C} @ \mathrm{G}-\mathrm{S}$.

Materials Characterization. The phase constituents were determined by XRD (Rigaku SmartLab $9 \mathrm{~kW}$ ). The morphology of the samples were investigated using scanning electron microscopy (SEM) (Thermofisher Apreo C) and transmission electron microscope (TEM) (Thermofisher Talos F200S). XPS analysis (ThermoFisher Escalab 250Xi) was performed to analyze the elemental composition and content. The UV-vis absorption spectra of polysulfides were measured by a spectrophotometer (UV-2600). The Raman spectra were collected using a Raman microscope (Renishaw inVia Qontor) system.

Electrochemical Measurements. The cathode was prepared by pasting a sulfur slurry using $N$-methylpyrrolidone as solvent (for instance, 80 wt \% 
$\mathrm{Bi} / \mathrm{Bi}_{2} \mathrm{O}_{3} @ \mathrm{C} @ \mathrm{G}-\mathrm{S}, 10$ wt \% CNTs, and 10 wt \% PVDF binder) onto the aluminum foil, subjected to vacuum-drying at $60{ }^{\circ} \mathrm{C}$ for $12 \mathrm{~h}$. The coin cells (CR2025) were assembled by using $\mathrm{Bi} / \mathrm{Bi}_{2} \mathrm{O}_{3} @ \mathrm{C} @ \mathrm{G}-\mathrm{S}$ as the cathode, lithium foil as the anode, $\mathrm{PP}$ as the separator, and $1 \mathrm{M}$ LiTFSI in 1,3-dioxolane (DOL)/dimethoxyethane (DME) (volume ratio of $1: 1$ ) as the electrolyte with $1 \mathrm{wt} \% \mathrm{LiNO}_{3}$ electrolyte additive. The amount of the electrolyte was controlled to $8-12 \mu \mathrm{mg}^{-1}$ sulfur. $\mathrm{CV}$ curves were recorded on an electrochemical workstation (Autolab M204) at a scanning rate of 0.1 $\mathrm{mV} \mathrm{s}^{-1}$ in the range between 2.8 and $1.7 \mathrm{~V}$ at room temperature. The galvanostatic discharge-charge cycle tests and rate tests were carried out on a Neware battery testing system at current densities of $0.2-3 \mathrm{C}\left(1 \mathrm{C}=1675 \mathrm{~mA} \mathrm{~g}^{-1}\right)$ with a cutoff voltage of $2.8-1.7 \mathrm{~V} v s$. $\mathrm{Li} / \mathrm{Li}^{+}$at room temperature. The specific capacity was calculated based on the mass of sulfur. Electrochemical impedance spectroscopy was measured in the charged state at $2.8 \mathrm{~V}$. The voltage a mplitude applied to the coin cells was $5 \mathrm{mV}$, with the frequency range from $100 \mathrm{kHz}$ to $1 \mathrm{~Hz}$.

Symmetrical Cell Assembly and Measurements. Symmetric cells were assembled using two identical electrodes $\left(\mathrm{Bi}_{2} \mathrm{O}_{3} @ \mathrm{C} @ \mathrm{G}, \mathrm{Bi} / \mathrm{Bi}_{2} \mathrm{O}_{3} @ \mathrm{C} @ \mathrm{G}\right.$, or Bi@C@G) with $20 \mu \mathrm{L}$ of $\mathrm{Li}_{2} \mathrm{~S}_{6}$ electrolyte. $\mathrm{S}$ and $\mathrm{Li}_{2} \mathrm{~S}$ powders at a molar ratio of $5: 1$ were added into the DOLDME solution (1:1 by volume) and stirred for $24 \mathrm{~h}$ under $60{ }^{\circ} \mathrm{C}$ to prepare the $\mathrm{Li}_{2} \mathrm{~S}_{6}$ solution. $\mathrm{CV}$ was carried out in the voltage range from -1.5 to $1.5 \mathrm{~V}$ at a scan rate of $10 \mathrm{mV} \mathrm{s}^{-1}$.

$\mathrm{Li}_{2} \mathrm{~S}$ Nucleation Experiments. Sulfur and $\mathrm{Li}_{2} \mathrm{~S}$ were stirred in a molar ratio of 7:1 in tetraglyme solvent for $24 \mathrm{~h}$ to obtain the $\mathrm{Li}_{2} \mathrm{~S}_{8}$ solution $\left(0.2 \mathrm{~mol} \mathrm{~L}{ }^{-1}\right) . \mathrm{Bi}_{2} \mathrm{O}_{3} @ \mathrm{C} @ \mathrm{G}$ 
@CP, Bi/Bi $\mathrm{O}_{3} @ \mathrm{C} @ \mathrm{G} @ \mathrm{CP}$, and $\mathrm{Bi} @ \mathrm{C} @ \mathrm{G} @ \mathrm{CP}$ were used as collectors, respectively. $25 \mu \mathrm{L}$ of $\mathrm{Li}_{2} \mathrm{~S}_{8}$ solution was first deposited onto the working electrode, and then $25 \mu \mathrm{L}$ of LiTFSI (1.0 mol L-1) without $\mathrm{Li}_{2} \mathrm{~S}_{8}$ was dropped onto the lithium anode. The batteries were discharged at $0.112 \mathrm{~mA}$ to $2.06 \mathrm{~V}$ and then kept potentiostatically at $2.05 \mathrm{~V}$ for $\mathrm{Li}_{2} \mathrm{~S}$ to nucleate and grow until the current fell below $0.01 \mathrm{~mA}$. The nucleation rate of $\mathrm{Li}_{2} \mathrm{~S}$ on the substrates was calculated based on Faraday's law.

Visualized Adsorption Test. A $5 \times 10^{-4} \mathrm{M} \mathrm{Li}_{2} \mathrm{~S}_{6}$ solution was prepared by adding sulfur and $\mathrm{Li}_{2} \mathrm{~S}$ at a molar ratio of 1:3 in 1,2-dimethoxymethane/1,3-dioxolane (DME/DOL) (1:1 by volume) solvent followed by vigorous magnetic stirring at room temperature until a uniform solution was obtained. 10 mg of $\mathrm{Bi}_{2} \mathrm{O}_{3} @ \mathrm{C} @ \mathrm{G}$, $\mathrm{Bi} / \mathrm{Bi}_{2} \mathrm{O}_{3} @ \mathrm{C} @ \mathrm{G}$, and $\mathrm{Bi} @ \mathrm{C} @ \mathrm{G}$ powders were added into $3 \mathrm{~mL}$ of the above solution, respectively, and aged to observe the color change. These suspensions were subjected to UV analysis as well.

In-situ Raman Spectroscopy Analysis. A LSB identical to the coin battery was assembled within in-situ Raman sample chamber (provided by Beijing Science Star technology Co., Ltd. China). The battery was continuously charged/discharged at current rate of $0.2 \mathrm{C}$ while the Raman spectrum was simultaneously recorded every 10 $\min$.

In-situ UV-vis Absorption Spectra. In-situ UV-vis spectroscopic measurements were performed on a spectrophotometer (UV-2600). The spectro-electrochemical cell used in the measurement was described in our previous work. ${ }^{1}$ The $\mathrm{Bi}_{2} \mathrm{O}_{3} @ \mathrm{C} @ \mathrm{G}$, Bi/ $/ i_{2} \mathrm{O}_{3} @ \mathrm{C} @ \mathrm{G}$, or Bi@C@G-coated glassy carbon electrode was used as cathode, 
with platinum wire as anode. $0.5 \mathrm{M} \mathrm{Li}_{2} \mathrm{~S}_{8}$ in dimethyl sulfoxide was used as the electrolyte. During the tests, the potential was controlled by an electrochemical workstation (Autolab M204). Here, the UV-vis absorbance was normalized with the absorbance at $2.8 \mathrm{~V}$.

Computation. We have employed the first-principles ${ }^{2-3}$ to perform all Spin-polarization density functional theory (DFT) calculations within the generalized gradient approximation (GGA) using the Perdew-Burke-Ernzerhof (PBE) formulation. ${ }^{4}$ The projected augmented wave (PAW) potentials were chosen to describe the ionic cores and take valence electrons into account, using a plane wave basis set with a kinetic energy cutoff of $450 \mathrm{eV}$. Partial occupancies of the Kohn-Sham orbitals were allowed using the Gaussian smearing method and a width of $0.05 \mathrm{eV}$. The electronic energy was considered self-consistent when the energy change was smaller than $10^{-6} \mathrm{eV}$. A geometry optimization was considered convergent when the energy change was smaller than $0.04 \mathrm{eV}^{-1}$. The vacuum spacing in a direction perpendicular to the plane of the structure is $15 \AA$ for $\mathrm{Bi}(104)$ and $\mathrm{Bi}_{2} \mathrm{O}_{3}$ (201) surface. The Brillouin zone integration was performed using $3 \times 3 \times 1$ Monkhorst-Pack k-point sampling for a structure. Finally, the adsorption energies $\left(E_{a d s}\right)$ were calculated as $E_{a d s}=E_{a d / s u b}-E_{a d}-E_{s u b}$, where $E_{a d / s u b}, E_{a d}$, and $E_{\text {sub }}$ are the total energies of the optimized adsorbate/substrate system, the adsorbate in the structure, and the clean substrate, respectively. The free energy was calculated using the equation:

$$
G=E+Z P E-T S
$$


where G, E, ZPE and TS are the free energy, total energy from DFT calculations, zero point energy and entropic contributions, respectively.

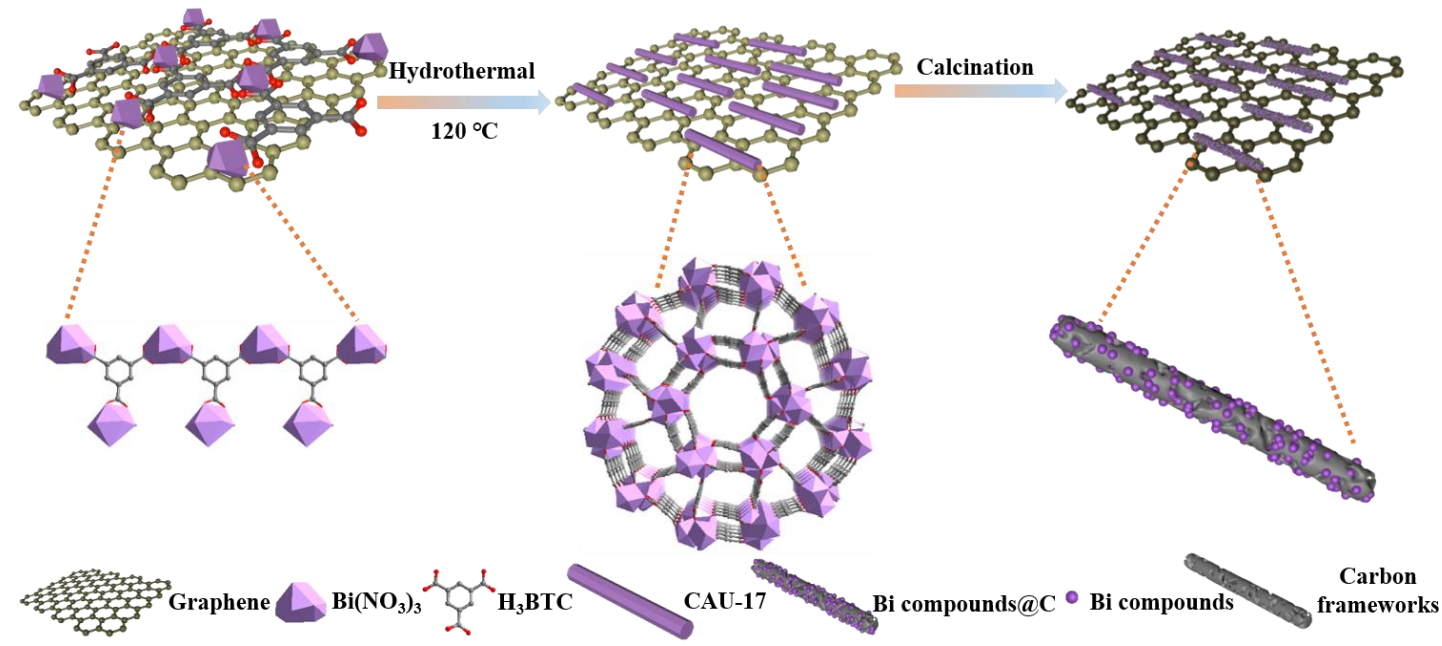

Figure S1. Schematic illustration to synthesize Bi compounds@C@G heterostructure.
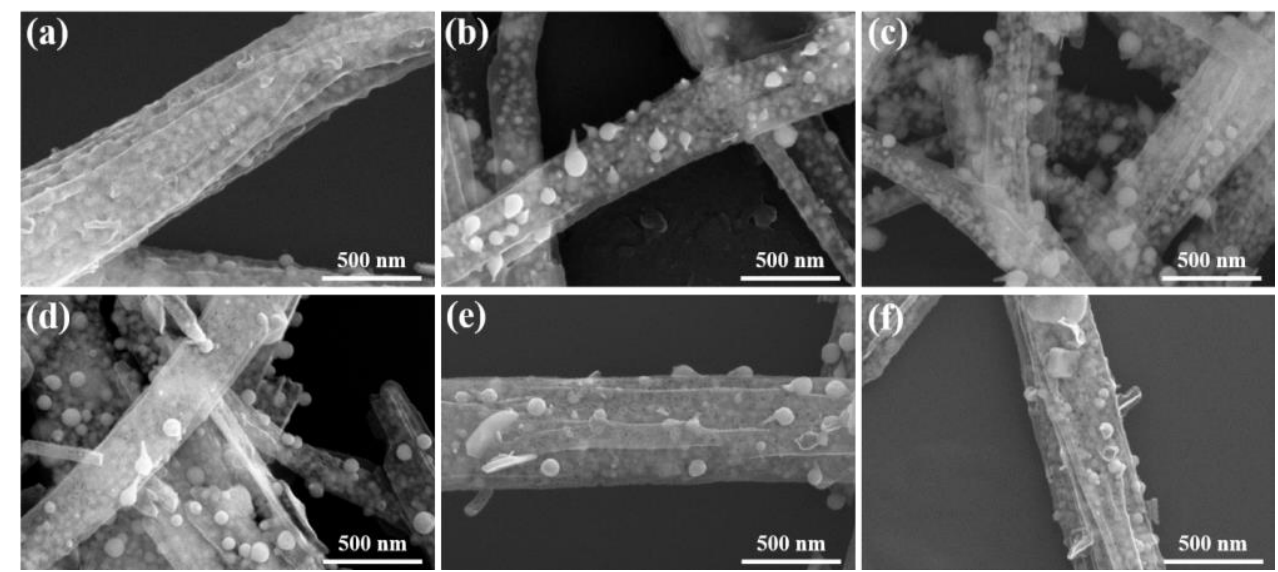

Figure S2. SEM images of the synthesized $\mathrm{Bi}_{2} \mathrm{O}_{3} @ \mathrm{C}-400$ (a), $\mathrm{Bi}_{2} \mathrm{O}_{3} @ \mathrm{C}-500$ (b), $\mathrm{Bi}_{2} \mathrm{O}_{3} @ \mathrm{C}-600$ (c), $\mathrm{Bi}_{2} \mathrm{O}_{3} @ \mathrm{C}-700$ (d), $\mathrm{Bi} \mathrm{Bi}_{2} \mathrm{O}_{3} @ \mathrm{C}-800$ (e), and $\mathrm{Bi} @ \mathrm{C}-900$ (f). 


\section{$500 \mathrm{~nm}$}

Figure S3. SEM images of the synthesized $\mathrm{Bi} / \mathrm{Bi}_{2} \mathrm{O}_{3} @ \mathrm{C}-800$ (enlarged of Figure S2e).

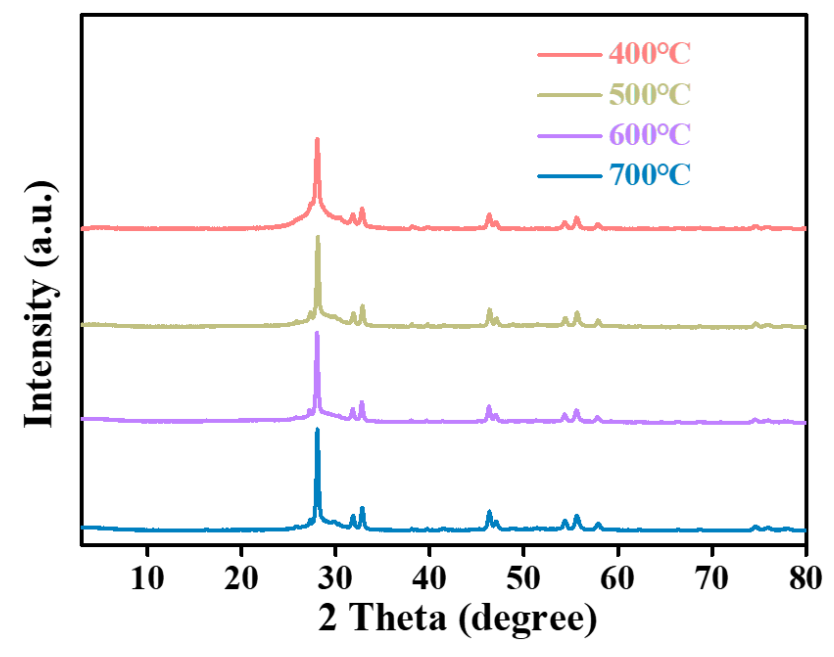

Figure S4. PXRD patterns of $\mathrm{Bi}_{2} \mathrm{O}_{3} @ \mathrm{C}-400, \mathrm{Bi}_{2} \mathrm{O}_{3} @ \mathrm{C}-500$ (b), $\mathrm{Bi}_{2} \mathrm{O}_{3} @ \mathrm{C}-600$ (c), and $\mathrm{Bi}_{2} \mathrm{O}_{3} @ \mathrm{C}-700(\mathrm{~d})$. 


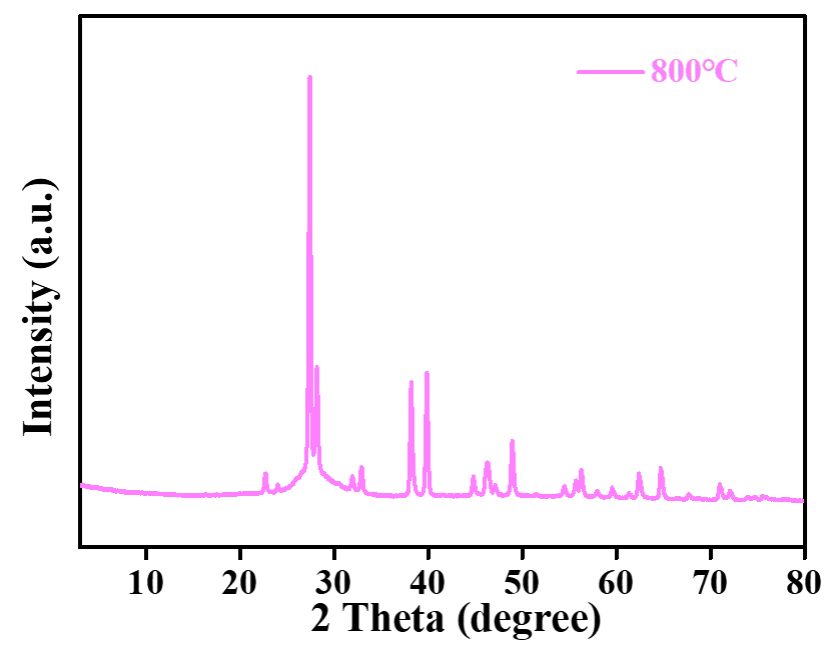

Figure S5. PXRD patterns of $\mathrm{Bi} / \mathrm{Bi}_{2} \mathrm{O}_{3} @ \mathrm{C}-800$.

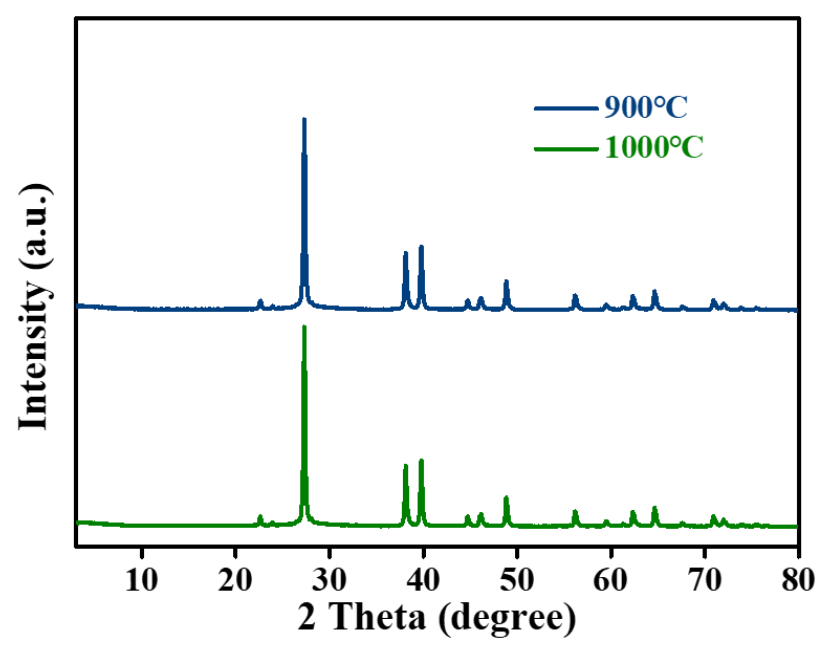

Figure S6. PXRD patterns of Bi@C-900 and Bi@C-1000.
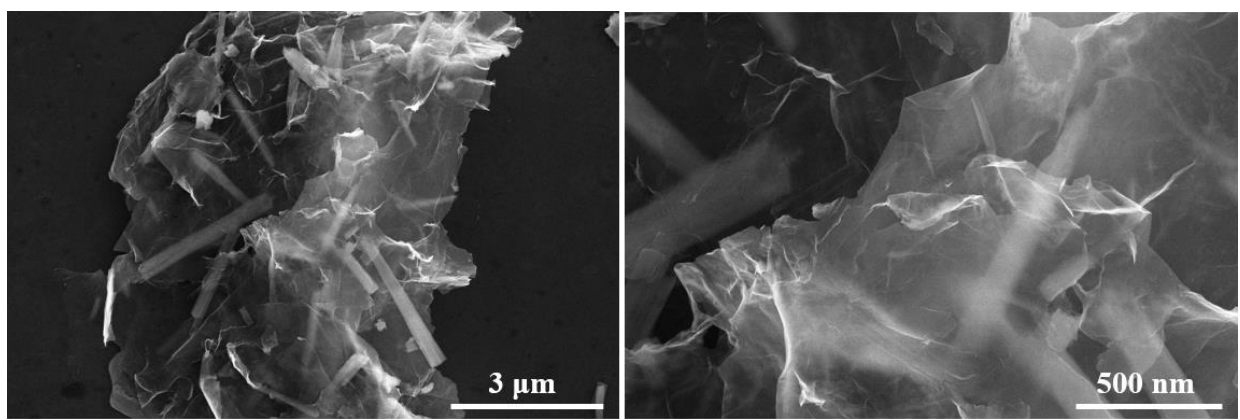

Figure S7. SEM images of the synthesized Bi-MOF@G. 

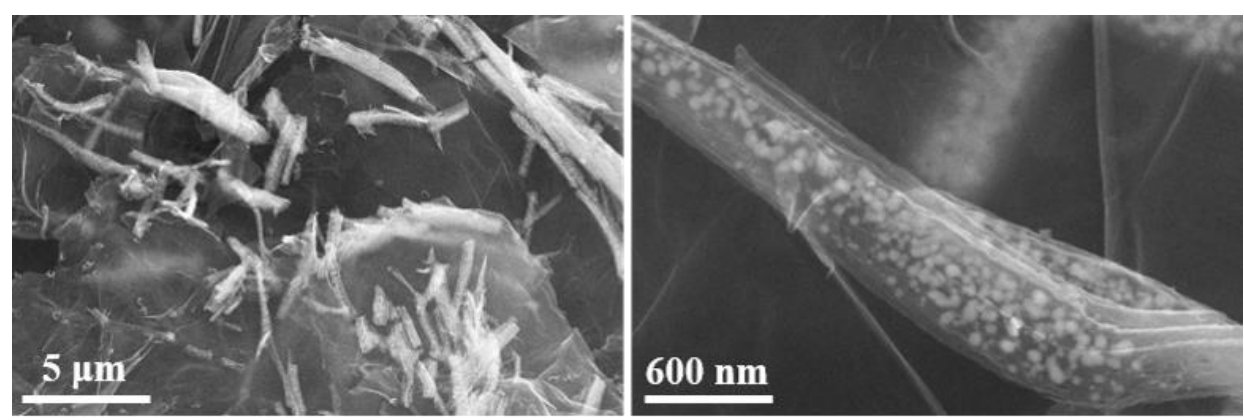

Figure S8. SEM images of the synthesized $\mathrm{Bi} / \mathrm{Bi}_{2} \mathrm{O}_{3} @ \mathrm{C} @ \mathrm{G}$.
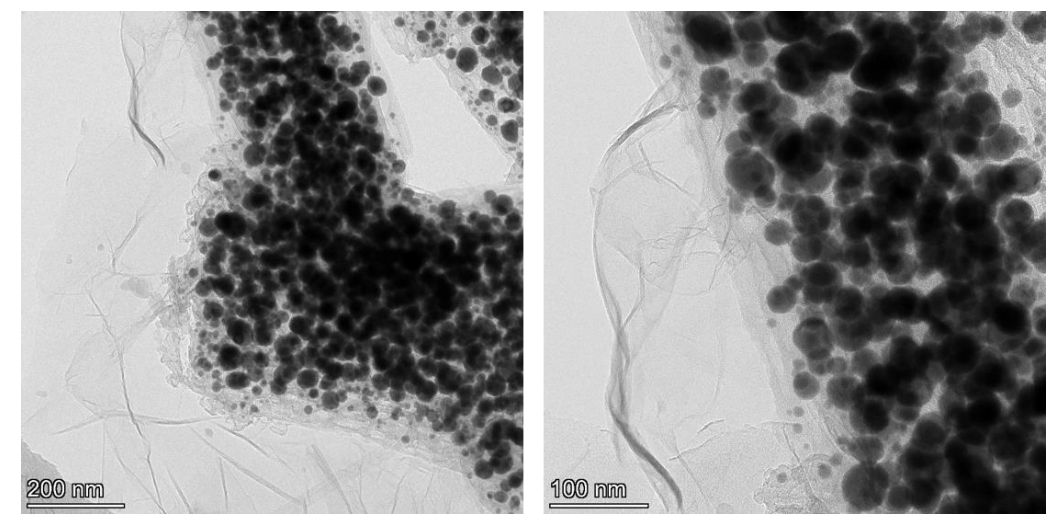

Figure S9. TEM images of the synthesized $\mathrm{Bi}_{2} \mathrm{O}_{3} @ \mathrm{C} @ \mathrm{G}$.
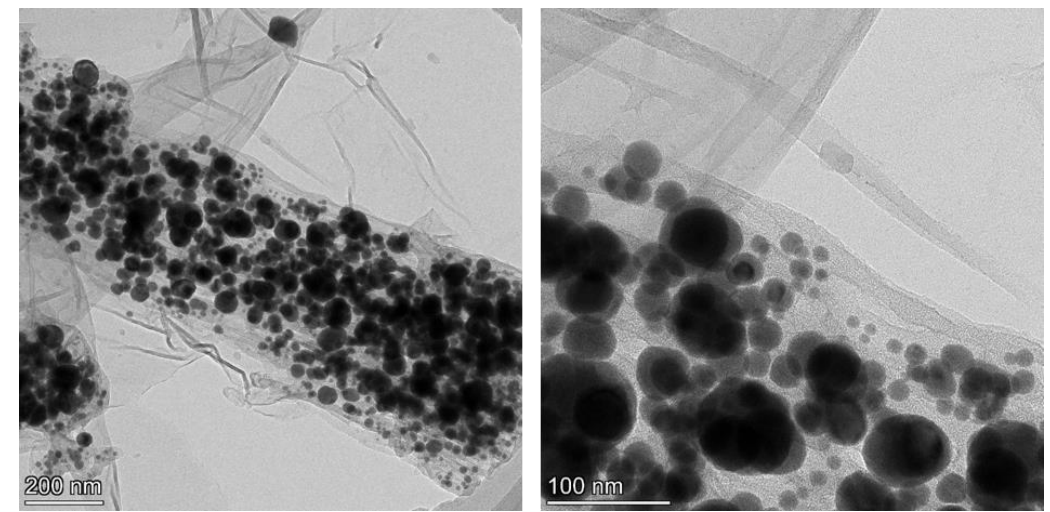

Figure S10. TEM images of the synthesized Bi@C@G. 


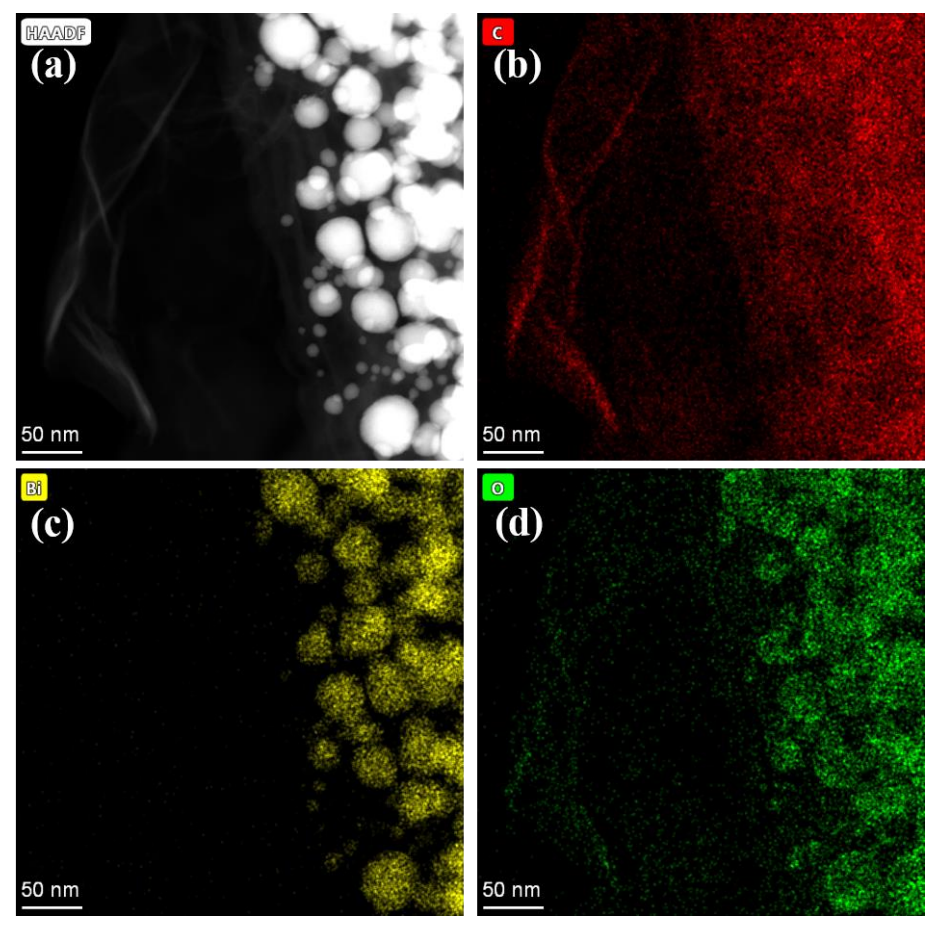

Figure S11. TEM elemental mapping images of the synthesized $\mathrm{Bi}_{2} \mathrm{O}_{3} @ \mathrm{C} @ \mathrm{G}$.

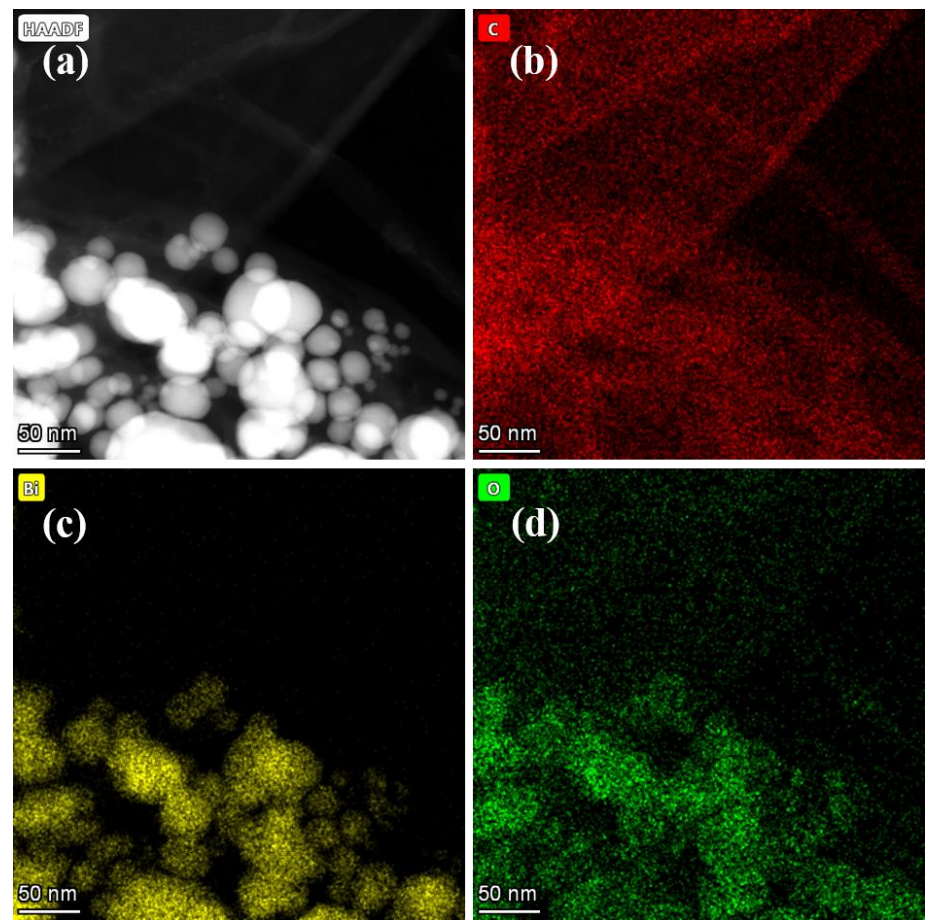

Figure S12. TEM elemental mapping images of the synthesized $\mathrm{Bi} \mathrm{C} @ \mathrm{G}$. 


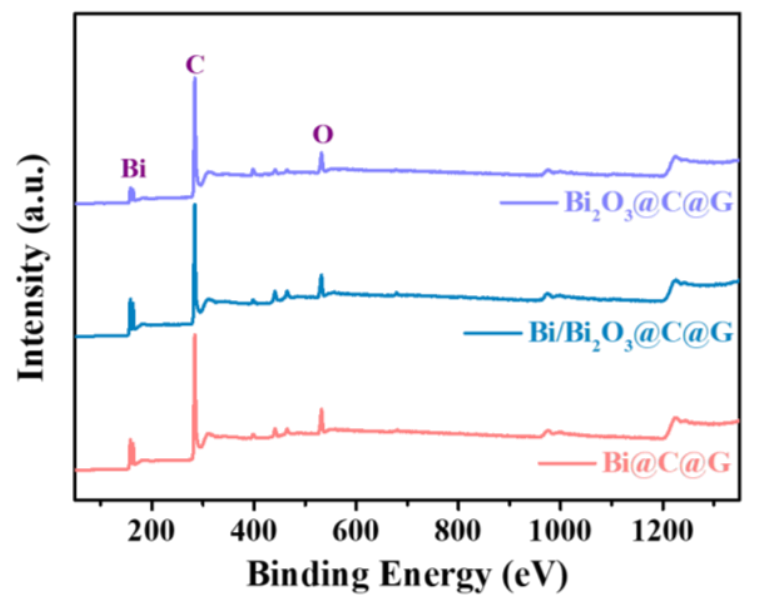

Figure S13. XPS survey spectra of $\mathrm{Bi}_{2} \mathrm{O}_{3} @ \mathrm{C} @ \mathrm{G}, \mathrm{Bi} / \mathrm{Bi}_{2} \mathrm{O}_{3} @ \mathrm{C} @ \mathrm{G}$, and $\mathrm{Bi} @ \mathrm{C} @ \mathrm{G}$.
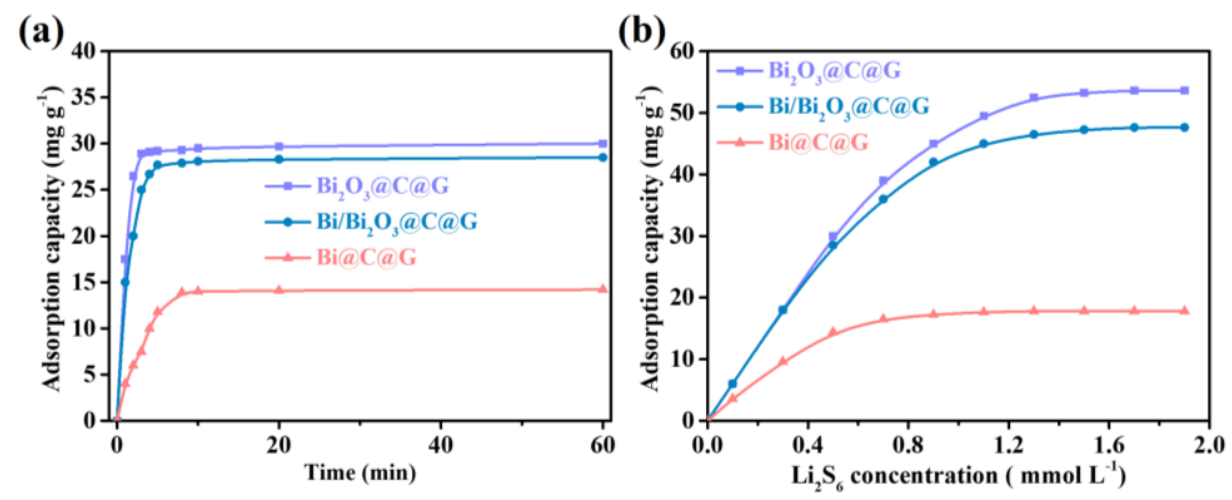

Figure S14. Comparison of the adsorption kinetics (a) and saturate adsorption capacities (b) of $\mathrm{Bi}_{2} \mathrm{O}_{3} @ \mathrm{C} @ \mathrm{G}, \mathrm{Bi} / \mathrm{Bi}_{2} \mathrm{O}_{3} @ \mathrm{C} @ \mathrm{G}$ and $\mathrm{Bi} @ \mathrm{C} @ \mathrm{G}$ for $\mathrm{Li}_{2} \mathrm{~S}_{6}$.
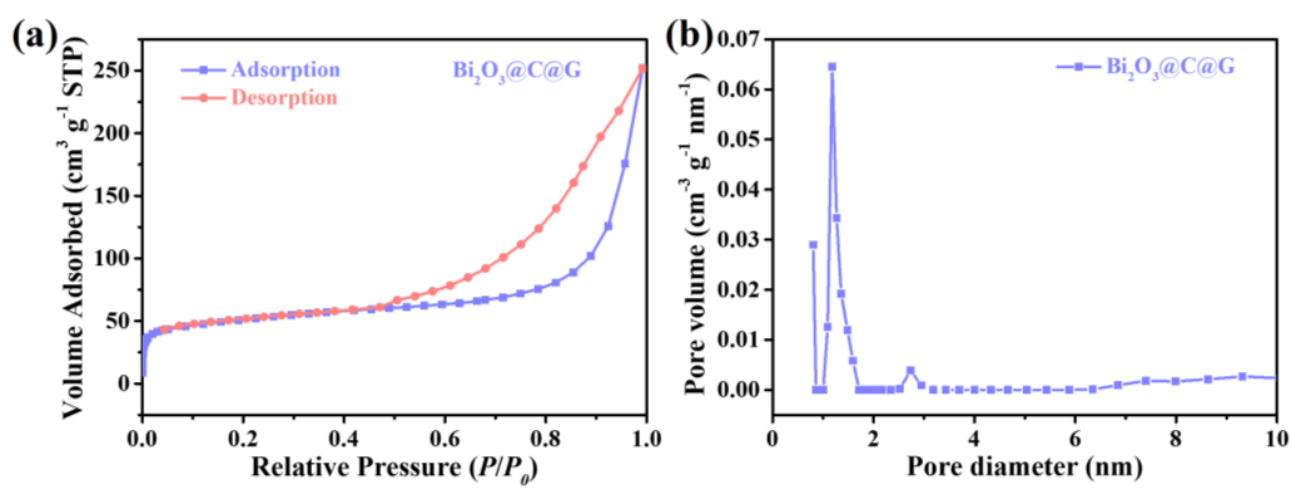

Figure S15. Nitrogen adsorption/desorption isotherms (a) and pore size distribution curves (b) of $\mathrm{Bi}_{2} \mathrm{O}_{3} @ \mathrm{C} @ \mathrm{G}$. 

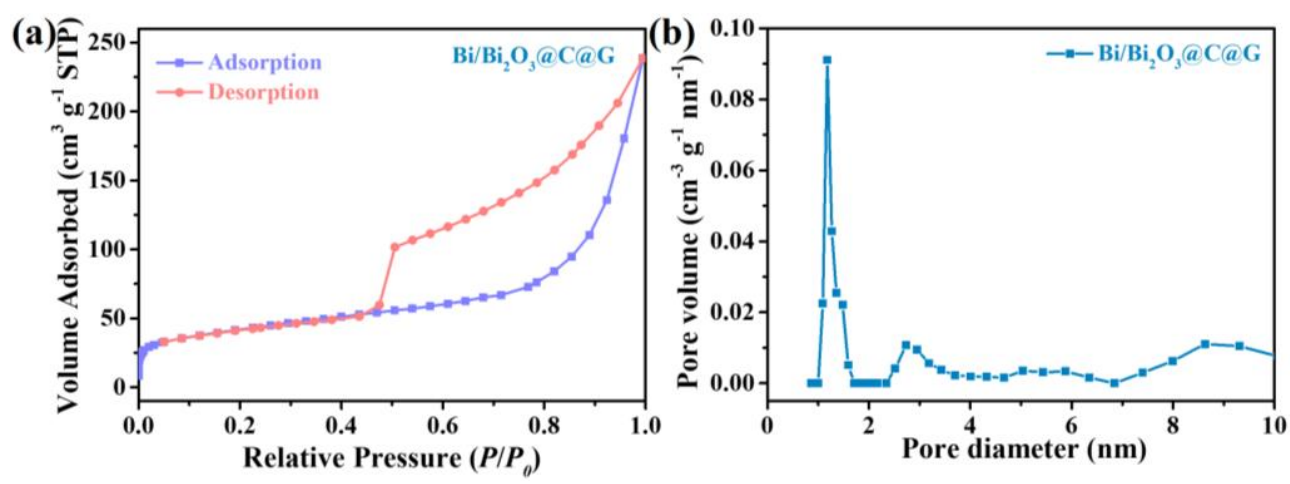

Figure S16. Nitrogen adsorption/desorption isotherms (a) and pore size distribution curves (b) of $\mathrm{Bi} / \mathrm{Bi}_{2} \mathrm{O}_{3} @ \mathrm{C} @ \mathrm{G}$.
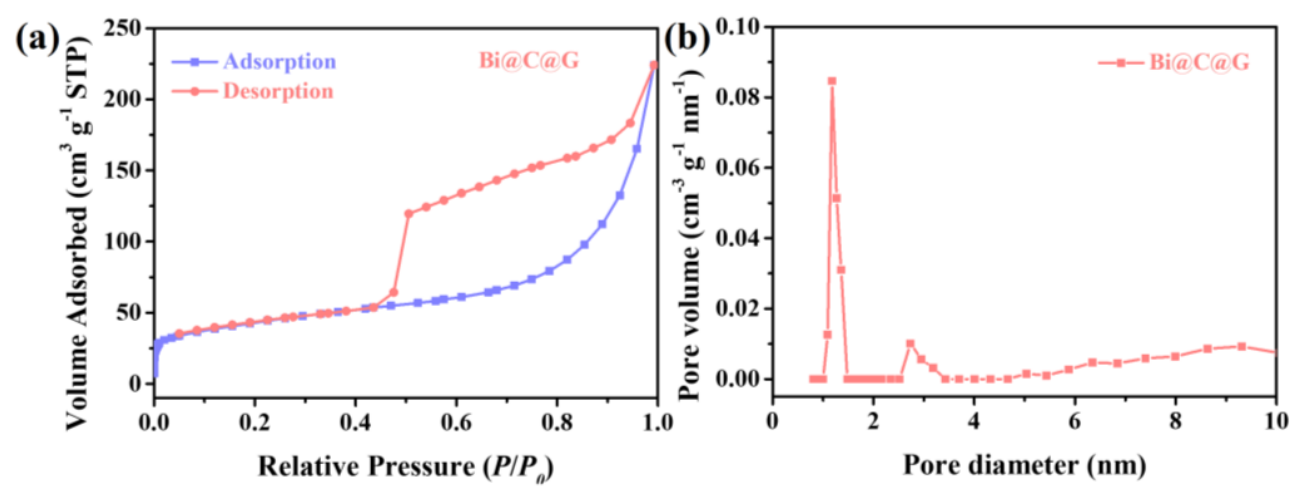

Figure S17. Nitrogen adsorption/desorption isotherms (a) and pore size distribution curves (b) of Bi@C@G.
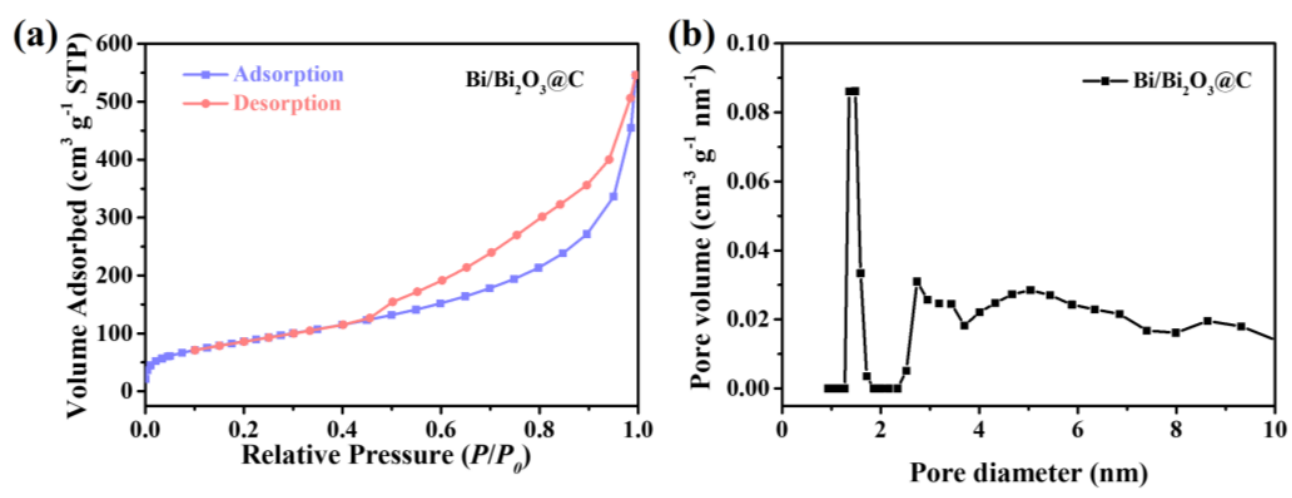

Figure S18. Nitrogen adsorption/desorption isotherms (a) and pore size distribution

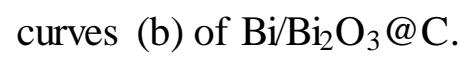



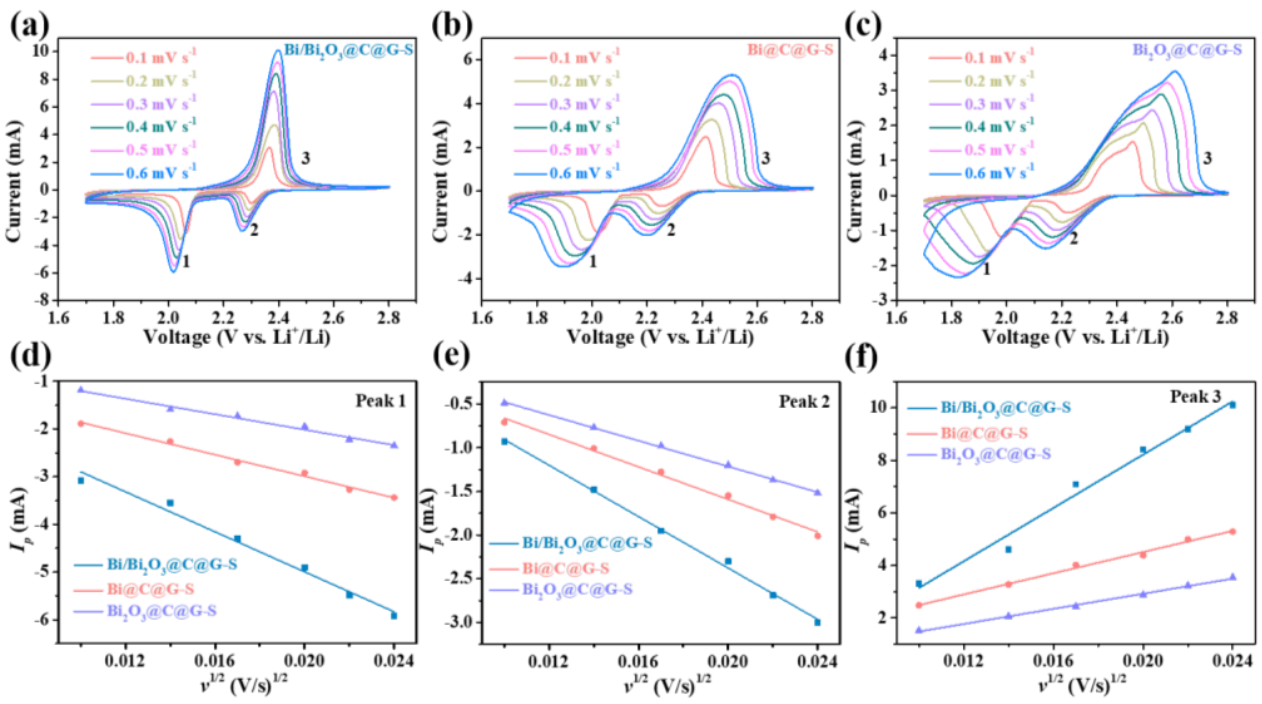

Figure S19. CV curves of $\mathrm{Bi} / \mathrm{Bi}_{2} \mathrm{O}_{3} @ \mathrm{C} @ \mathrm{G}-\mathrm{S}$ (a), $\mathrm{Bi} @ \mathrm{C} @ \mathrm{G}-\mathrm{S}$ (b), and

$\mathrm{Bi}_{2} \mathrm{O}_{3} @ \mathrm{C} @ \mathrm{G}-\mathrm{S}$ (c) cathodes at scan rates of $0.1,0.2,0.3,0.4,0.5$ and $0.6 \mathrm{mV} \mathrm{s}{ }^{-1}$. (d-f)

The plots of Ip- $v^{1 / 2}$ extracted from the peak currents in (a-c) CV curves.

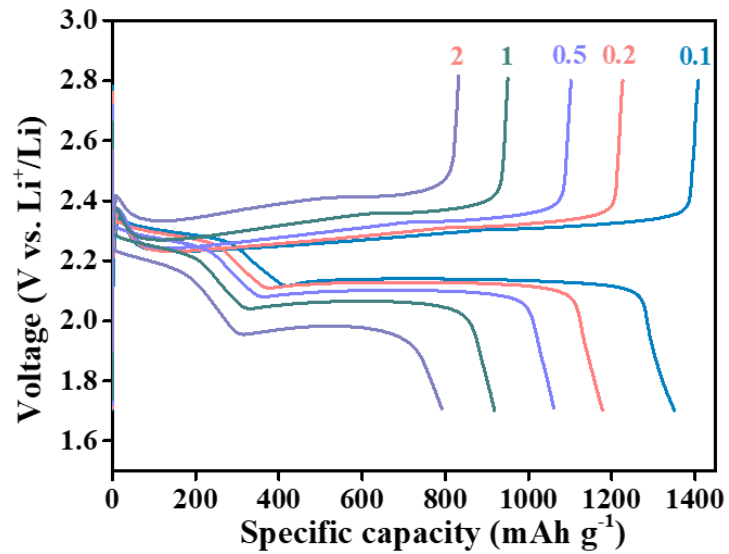

Figure S20. Galvanostatic charge-discharge curves of $\mathrm{Bi} / \mathrm{Bi}_{2} \mathrm{O}_{3} @ \mathrm{C} @ \mathrm{G}-\mathrm{S}$ at $0.1-2 \mathrm{C}$.

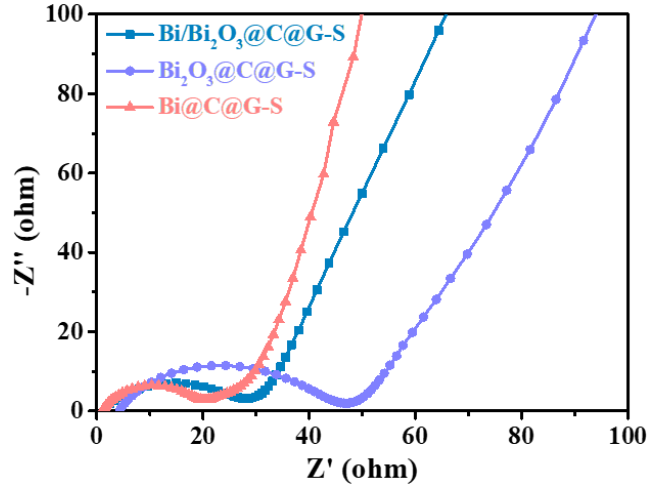

Figure S21. Nyquist plots of $\mathrm{Bi} / \mathrm{Bi}_{2} \mathrm{O}_{3} @ \mathrm{C} @ \mathrm{G}-\mathrm{S}, \mathrm{Bi} @ \mathrm{C} @ \mathrm{G}-\mathrm{S}$, and $\mathrm{Bi}_{2} \mathrm{O}_{3} @ \mathrm{C} @ \mathrm{G}-\mathrm{S}$ cathodes before cycling. 


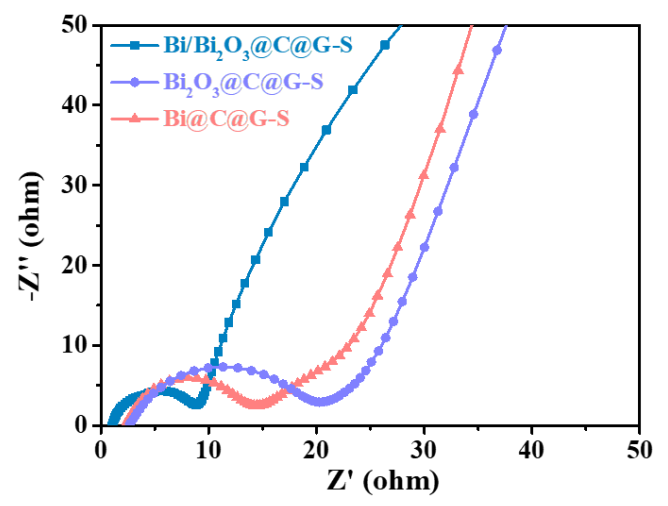

Figure S22. Nyquist plots of $\mathrm{Bi} / \mathrm{Bi}_{2} \mathrm{O}_{3} @ \mathrm{C} @ \mathrm{G}-\mathrm{S}$, Bi@C@G-S, and $\mathrm{Bi}_{2} \mathrm{O}_{3} @ \mathrm{C} @ \mathrm{G}-\mathrm{S}$ cathodes after 200 cycles at $0.2 \mathrm{C}$.

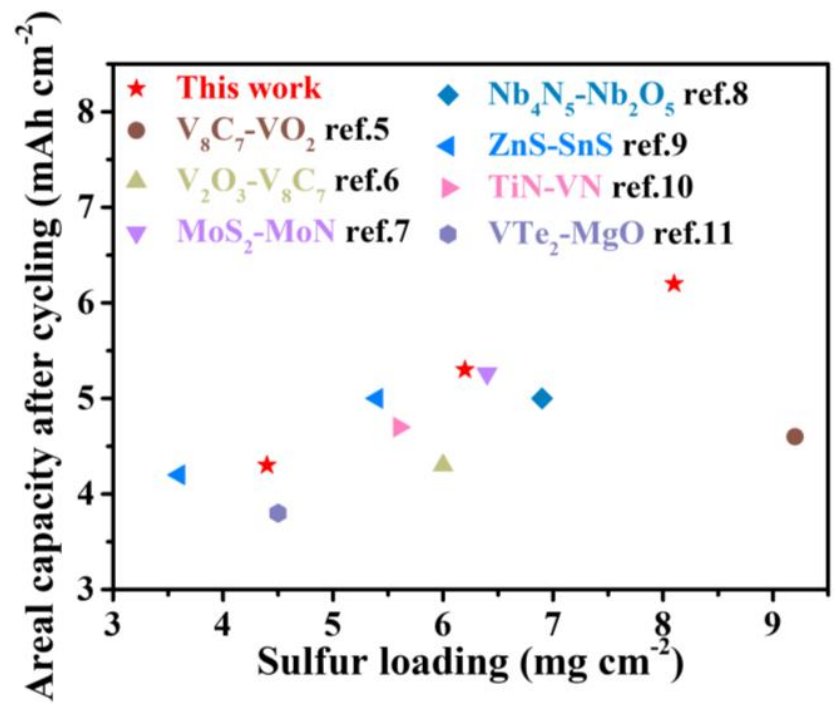

Figure S23. Comparison of areal capacities of high-loading cathodes between this work and other reported sulfur hosts. ${ }^{5-11}$

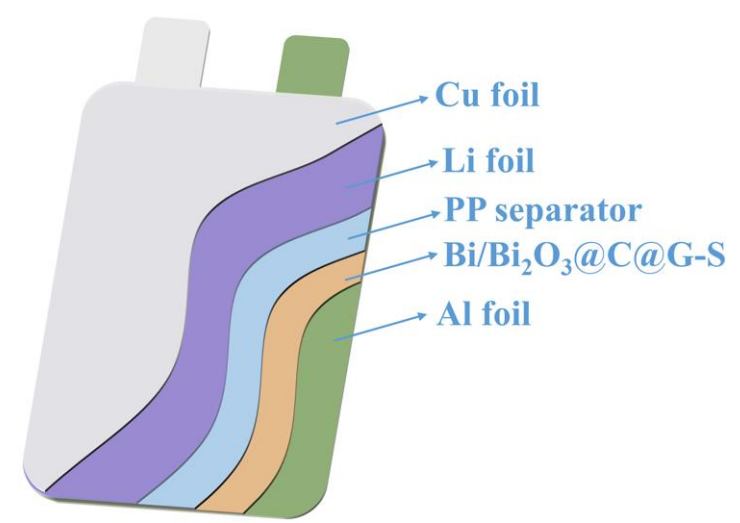

Figure S24. Schematic illustration of the pouch cell with $\mathrm{Bi}_{-} \mathrm{Bi}_{2} \mathrm{O}_{3} @ \mathrm{C} @ \mathrm{G}-\mathrm{S}$ cathode. 

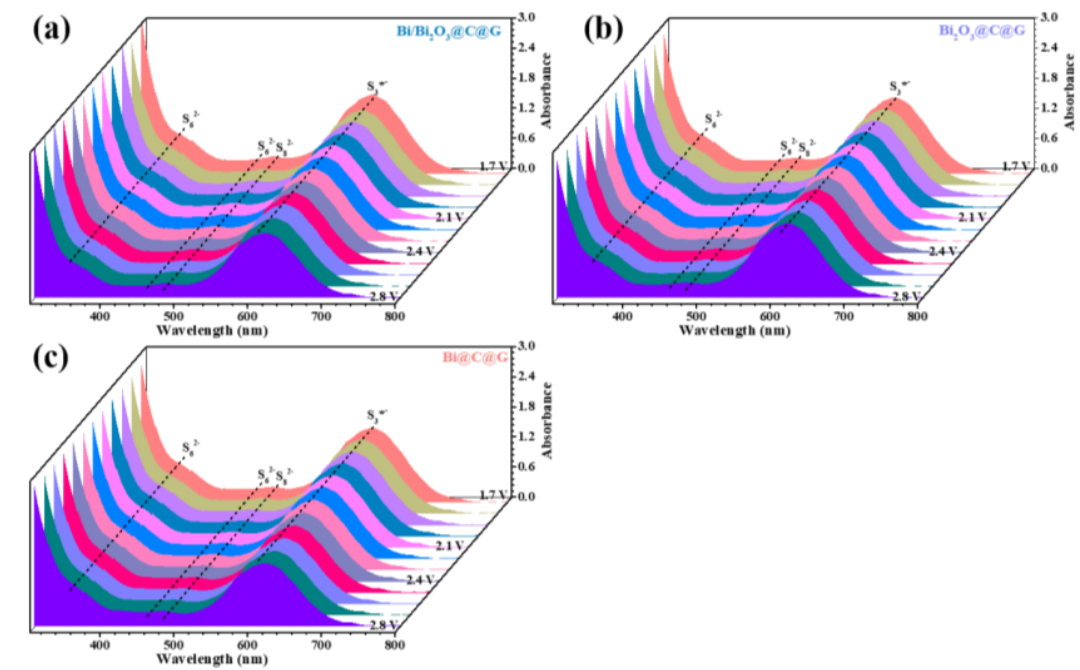

Figure S25. In-situ UV-vis spectra of $\mathrm{Li}_{2} \mathrm{~S}_{8}$ solution during discharge process with Bi/ $/ \mathrm{Bi}_{2} \mathrm{O}_{3} @ \mathrm{C} @ \mathrm{G}(\mathrm{a}), \mathrm{Bi}_{2} \mathrm{O}_{3} @ \mathrm{C} @ \mathrm{G}(\mathrm{b})$ and $\mathrm{Bi} @ \mathrm{C} @ \mathrm{G}-\mathrm{S}$ (c) as electrodes.
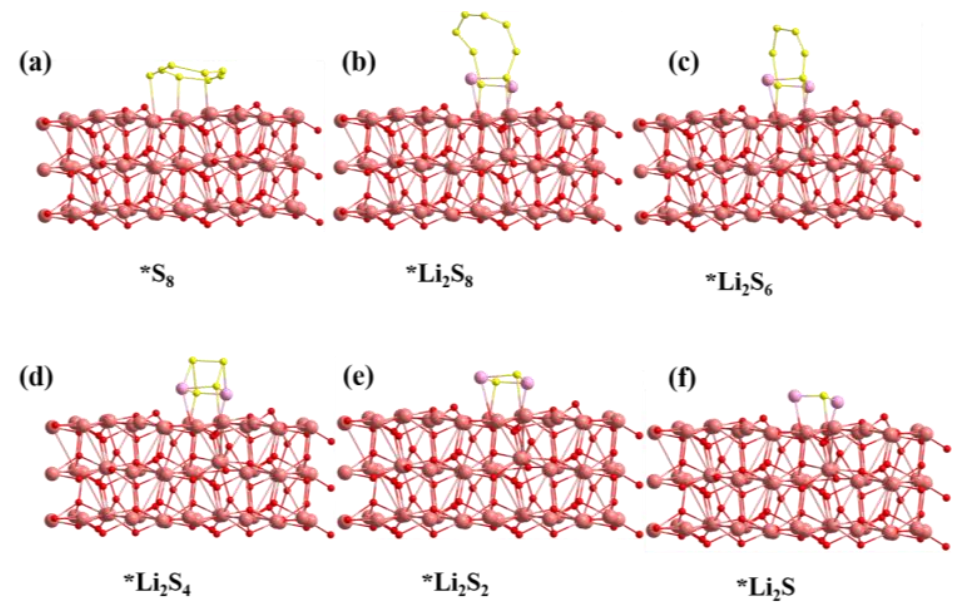

Figure S26. Calculated adsorption energies between $\mathrm{S}_{8}(\mathrm{a}), \mathrm{Li}_{2} \mathrm{~S}_{8}(\mathrm{~b}), \mathrm{Li}_{2} \mathrm{~S}_{6}(\mathrm{c}), \mathrm{Li}_{2} \mathrm{~S}_{4}$ (d), $\mathrm{Li}_{2} \mathrm{~S}_{2}(\mathrm{e}), \mathrm{Li}_{2} \mathrm{~S}(\mathrm{f})$ and $\mathrm{Bi}_{2} \mathrm{O}_{3}$. 
(a)

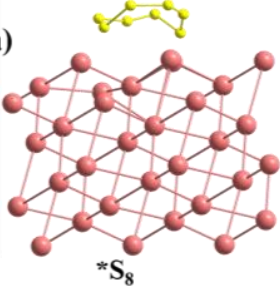

(d)

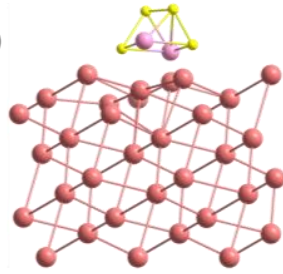

${ }^{*} \mathrm{Li}_{2} \mathrm{~S}_{4}$ (b)

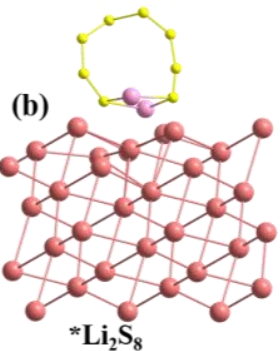

(e)

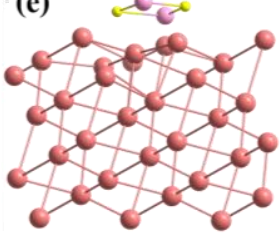

${ }^{*} \mathrm{Li}_{2} \mathrm{~S}_{2}$
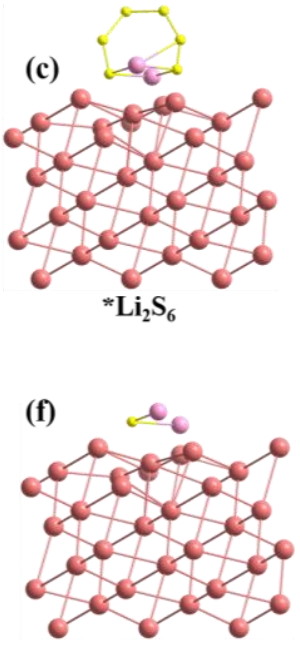

${ }^{*} \mathrm{Li}_{2} \mathrm{~S}$

Figure S27. Calculated adsorption energies between $\mathrm{S}_{8}$ (a), $\mathrm{Li}_{2} \mathrm{~S}_{8}$ (b), $\mathrm{Li}_{2} \mathrm{~S}_{6}$ (c), $\mathrm{Li}_{2} \mathrm{~S}_{4}$

(d), $\mathrm{Li}_{2} \mathrm{~S}_{2}(\mathrm{e}), \mathrm{Li}_{2} \mathrm{~S}$ (f) and $\mathrm{Bi}$.

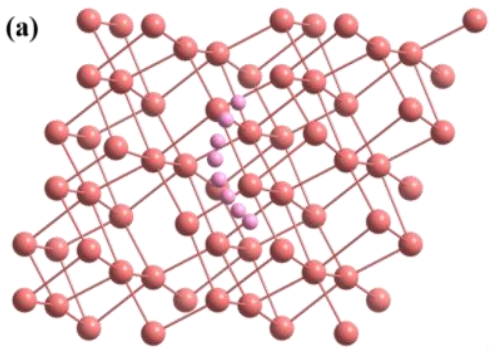

(b)

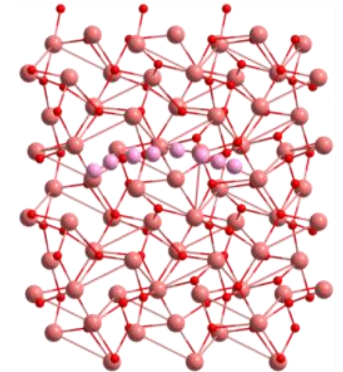

Figure S28. $\mathrm{Li}^{+}$diffusion path on $\mathrm{Bi}(\mathrm{a})$ and $\mathrm{Bi}_{2} \mathrm{O}_{3}(\mathrm{~b})$.

(a)

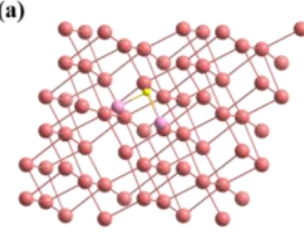

(b)

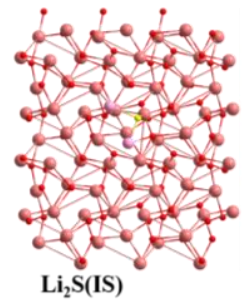

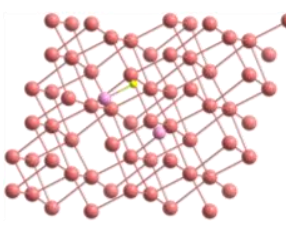

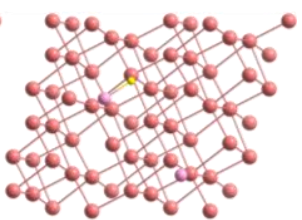

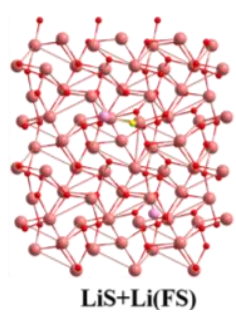

Figure 29. $\mathrm{Li}_{2} \mathrm{~S}$ decomposition processes on $\mathrm{Bi}(\mathrm{a})$ and $\mathrm{Bi}_{2} \mathrm{O}_{3}(\mathrm{~b})$. 
Table S1. Comparison of the key figures of merit of LSBs using our cathode with other state-of-the-art heterostructure catalysts cathodes.

\begin{tabular}{|c|c|c|c|c|c|}
\hline Catalysts & $\begin{array}{l}\text { Initial capacity } \\
\qquad\left[\mathrm{mA} \mathrm{h} \mathbf{g}^{-1}\right]\end{array}$ & $\begin{array}{l}\text { Cycle number } \\
\text { (retention) }\end{array}$ & Rate & $\begin{array}{c}\text { Decay } \\
\text { rate/cycle }\end{array}$ & Ref. \\
\hline $\mathrm{V}_{8} \mathrm{C}_{7} / \mathrm{VO}_{2}$ & 1022 & - & $1 \mathrm{C}$ & - & 5 \\
\hline TiN/VN & 986 & - & $1 \mathrm{C}$ & - & 6 \\
\hline $\mathrm{VTe}_{2} / \mathrm{MgO}$ & 927 & $1000(45 \%)$ & $1 \mathrm{C}$ & $0.055 \%$ & 7 \\
\hline $\mathrm{V}_{2} \mathrm{O}_{3} / \mathrm{V}_{8} \mathrm{C}_{7}$ & 877 & $1000(83 \%)$ & $1 \mathrm{C}$ & $0.017 \%$ & 8 \\
\hline $\mathrm{MoS}_{2} / \mathrm{MoN}$ & 865 & $1000(61 \%)$ & $1 \mathrm{C}$ & $0.039 \%$ & 9 \\
\hline $\mathrm{TiO}_{2} / \mathrm{TiN}$ & 610 & $2000(115.4 \%)$ & $1 \mathrm{C}$ & - & 12 \\
\hline $\mathrm{MoN} / \mathrm{VN}$ & 800 & $500(72.5 \%)$ & $1 \mathrm{C}$ & $0.05 \%$ & 13 \\
\hline $\mathrm{TiO}_{2} / \mathrm{Ni}_{3} \mathrm{~S}_{2}$ & 797 & - & $1 \mathrm{C}$ & - & 14 \\
\hline $\mathrm{NiO} / \mathrm{NiCO}_{2} \mathrm{O}_{4}$ & 1000 & $500(70.5 \%)$ & $0.5 \mathrm{C}$ & $0.059 \%$ & 15 \\
\hline $\mathrm{Co}_{9} \mathrm{~S}_{8} / \mathrm{CoO}$ & 925 & $1000(51 \%)$ & $1 \mathrm{C}$ & $0.049 \%$ & 16 \\
\hline $\mathrm{VO}_{2} / \mathrm{VN}$ & 1105 & - & $1 \mathrm{C}$ & - & 17 \\
\hline $\begin{array}{c}\mathrm{Bi}_{2} \mathrm{O}_{3} @ \mathrm{Co} / \mathrm{N}-\mathrm{PC} @ \\
\mathrm{~S} \\
\end{array}$ & 853 & $500(89.9 \%)$ & $1.5 \mathrm{C}$ & $0.0213 \%$ & 18 \\
\hline 2D-Bi & 1093 & $200(78 \%)$ & $0.5 \mathrm{C}$ & $0.11 \%$ & 19 \\
\hline $\mathrm{BiOCl} / \mathrm{G}-\mathrm{S}$ & 1230 & $200(78.9 \%)$ & $0.2 \mathrm{C}$ & $0.105 \%$ & 20 \\
\hline $\mathrm{Bi} / \mathrm{Bi}_{2} \mathrm{O}_{3}$ & 954 & $1000(77.5 \%)$ & $1 \mathrm{C}$ & $0.022 \%$ & $\begin{array}{r}\text { This } \\
\text { work }\end{array}$ \\
\hline
\end{tabular}




\section{REFERENCES}

(1) Zhou, S.; Yang, S.; Ding, X.; Lai, Y.; Nie, H.; Zhang, Y.; Chan, D.; Duan, H.; Huang, S.; Yang, Z. Dual-Regulation Strategy to Improve Anchoring and Conversion of Polysulfides in Lithium-Sulfur Batteries. ACS Nano 2020, 14, 7538-7551.

(2) Chadi, D. Special Points for Brillouin-Zone Integrations. Phys. Rev. B 1977, 16, 1746-1747.

(3) Perdew, J. P.; Burke, K.; Ernzerhof, M. Generalized Gradient Approximation Made Simple. Phys. Rev. Lett. 1996, 77, 3865-3868.

(4) Kresse, G.; Joubert, D. From Ultrasoft Pseudopotentials to the Projector Augmented-Wave Method. Phys. Rev. B 1999, 59, 1758-1775.

(5) Cai, J.; Jin, J.; Fan, Z.; Li, C.; Shi, Z.; Sun, J.; Liu, Z. 3D Printing of a $\mathrm{V}_{8} \mathrm{C}_{7}-\mathrm{VO}_{2}$ Bifunctional Scaffold as an Effective Polysulfide Immobilizer and Lithium Stabilizer for Li-S Batteries. Adv. Mater. 2020, 32, 2005967.

(6) Yao, Y.; Wang, H.; Yang, H.; Zeng, S.; Xu, R.; Liu, F.; Shi, P.; Feng, Y.; Wang, K.; Yang, W.; Wu, X.; Luo, W.; Yu, Y. A Dual-Functional Conductive Framework Embedded with TiN-VN Heterostructures for Highly Efficient Polysulfide and Lithium Regulation toward Stable Li-S Full Batteries. Adv. Mater. 2020, 32, 1905658. (7) Wang, M.; Song, Y.; Sun, Z.; Shao, Y.; Wei, C.; Xia, Z.; Tian, Z.; Liu, Z.; Sun, J. Conductive and Catalytic $\mathrm{VTe}_{2} @ \mathrm{MgO}$ Heterostructure as Effective Polysulfide Promotor for Lithium-Sulfur Batteries. ACS Nano 2019, 13, 13235-13243.

(8) Zhang, L.; Liu, Y.; Zhao, Z.; Jiang, P.; Zhang, T.; Li, M.; Pan, S.; Tang, T.; Wu, T.; Liu, P.; Hou, Y.; Lu, H. Enhanced Polysulfide Regulation via Porous Catalytic 
$\mathrm{V}_{2} \mathrm{O}_{3} / \mathrm{V}_{8} \mathrm{C}_{7}$ Heterostructures Derived from Metal-Organic Frameworks toward High-Performance Li-S Batteries. ACS Nano 2020, 14, 8495-8507.

(9) Wang, S.; Feng, S.; Liang, J.; Su, Q.; Zhao, F.; Song, H.; Zheng, M.; Sun, Q.;

Song, Z.; Jia, X.; Yang, J.; Li, Y.; Liao, J.; Li, R.; Sun, X. Insight into $\mathrm{MoS}_{2}-\mathrm{MoN}$ Heterostructure to Accelerate Polysulfide Conversion toward High-Energy-Density Lithium-Sulfur Batteries. Adv. Energy Mater. 2021, 11, 2003314.

(10) Shi, H.; Qin, J.; Lu, P.; Dong, C.; He, J.; Chou, X.; Das, P.; Wang, J.; Zhang, L.;

Wu, Z. Interfacial Engineering of Bifunctional Niobium (V)-Based Heterostructure Nanosheet toward High Efficiency Lean-Electrolyte Lithium-Sulfur Full Batteries. Adv. Funct. Mater. 2021, 31, 2102314.

(11) Yao, W.; Zheng, W.; Xu, J.; Tian, C.; Han, K.; Sun, W.; Xiao, S. ZnS-SnS@ NC Heterostructure as Robust Lithiophilicity and Sulfiphilicity Mediator toward High-Rate and Long-Life Lithium-Sulfur Batteries. ACS Nano 2021, 15, 7114-7130.

(12) Zhou, T.; Lv, W.; Li, J.; Zhou, G.; Zhao, Y.; Fan, S.; Liu, B.; Li, B.; Kang, F.; Yang, Q. Twinborn $\mathrm{TiO}_{2}$-TiN Heterostructures Enabling Smooth Trapping-Diffusion-Conversion of Polysulfides towards Ultralong Life Lithium-Sulfur Batteries. Energy. Environ. Sci. 2017, 10, 1694-1703.

(13) Ye, C.; Jiao, Y.; Jin, H.; Slattery, A.; Davey, K.; Wang, H.; Qiao, S. 2D MoN-VN Heterostructure to Regulate Polysulfides for Highly Efficient Lithium-Sulfur Batteries. Angew. Chem., Int. Ed. 2018, 57, 16703-16707. 
(14) Wang, R.; Luo, C.; Wang, T.; Zhou, G.; Deng, Y.; He, Y.; Zhang, Q.; Kang, F.; Lv, W.; Yang, Q. Bidirectional Catalysts for Liquid-Solid Redox Conversion in Lithium-Sulfur Batteries. Adv. Mater. 2020, 32, 2000315.

(15) Hu, L.; Dai, C.; Liu, H.; Li, Y.; Shen, B.; Chen, Y.; Bao, S.; Xu, M. Double-Shelled NiO-NiCo $2 \mathrm{O}_{4}$ Heterostructure@Carbon Hollow Nanocages as an Efficient Sulfur Host for Advanced Lithium-Sulfur Batteries. Adv. Energy Mater. 2018, $8,1800709$.

(16) Wang, N.; Chen, B.; Qin, K.; Liu, E.; Shi, C.; He, C.; Zhao, N. Rational Design of $\mathrm{Co}_{9} \mathrm{~S}_{8} / \mathrm{CoO}$ Heterostructures with Well-Defined Interfaces for Lithium Sulfur Batteries: A Study of Synergistic Adsorption-Electrocatalysis Function. Nano Energy 2019, 60, 332-339.

(17) Song, Y.; Zhao, W.; Kong, L.; Zhang, L.; Zhu, X.; Shao, Y.; Ding, F.; Zhang, Q.; Sun, J.; Liu, Z. Synchronous Immobilization and Conversion of Polysulfides on a $\mathrm{VO}_{2}-\mathrm{VN}$ Binary Host Targeting High Sulfur Load Li-S Batteries. Energy. Environ. Sci. 2018, 11, 2620-2630.

(18) Liu, H.; Chen, Z.; Zhou, L.; Li, X.; Pei, K.; Zhang, J.; Song, Y.; Fang, F.; Che, R.; Sun, D. Rooting Bismuth Oxide Nanosheets into Porous Carbon Nanoboxes as a Sulfur Immobilizer for Lithium-Sulfur Batteries. J. Mater. Chem. A 2019, 7, 7074-7081.

(19) $\mathrm{Xu}, \mathrm{H}$; ; Yang, S.; Li, B. Ultrathin Bismuth Nanosheets as an Efficient Polysulfide Catalyst for High Performance Lithium-Sulfur Batteries. J. Mater. Chem. A 2020, 8, 149-157. 
(20) Wu, X.; Liu, N.; Wang, M.; Qiu, Y.; Guan, B.; Tian, D.; Guo, Z.; Fan, L.; Zhang, N. A Class of Catalysts of $\mathrm{BiOX}(\mathrm{X}=\mathrm{Cl}, \mathrm{Br}$, I) for Anchoring Polysulfides and Accelerating Redox Reaction in Lithium Sulfur Batteries. ACS Nano 2019, 13, $13109-13115$. 